\title{
حصر بعض الأمراض الفطرية على أورلق الورد بمدينة البيضاء، ليبيا
}

\author{
نوارة علي محمد * نوال جمعة عبدالله وعمر موسي السنوسي

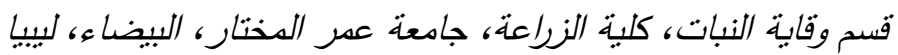

تاريخ الاستلام: 13 يونيو 2019 / تاريخ القبول: 30 ديسمبر 2019 https://doi.org/10.54172/mjsc.v34i4.235:Doi

المستخلص : أجريت هذه الدراسة على نباتات الورد النامية في مدينة البيضاء عام 2016 بهدف حصر الأمراض الفطرية على

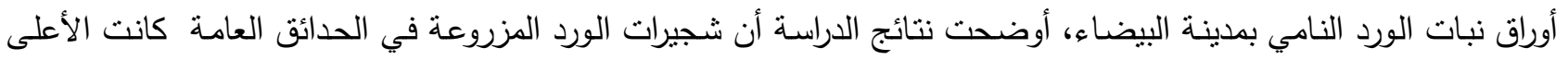

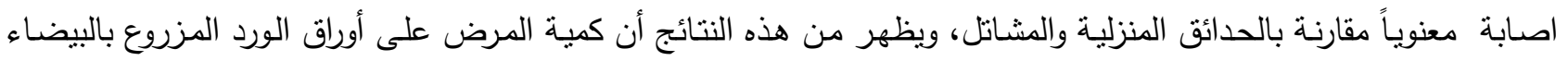

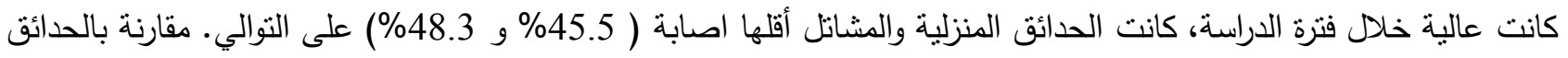
العامـة (73\%)،الأمراض التي سجلت في هذه الدراسـة كانت: البياض الدقيقي،الصدأ، البقعة السوداء، والبقعة الفضية، احتراق الحواف، واللطخة؛ أكثر الأمراض تواجداً هو مرض البياض الدقيقي حيث بلـغ (46.9\% و 30.4\%) لكل من نسبة الإصـابة وشدتها على أوراق الورد المزروعة بمدينة البيضاء على التوالي، بينما مرض الصدأ كان أقل الأمراض المسجلة بالمنطقة خلال

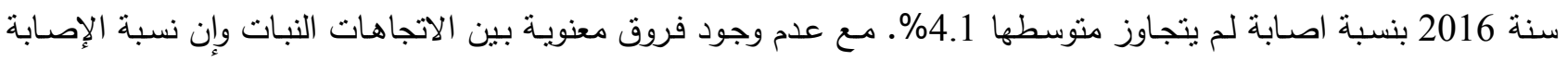
تتراوح بين 37.5-40\%، وكان شهر يوليو هو أعلى الثهور إصابة، وأن أوراق الورد المسنَّة هي الأعلى نسبة أصابة في جميع شهور الدراسة.

الكلمات المفتاحية: حصر ، أمراض فطرية، أوراق الورد، البيضاء، ليبيا

التجارية، هذه الأمراض تسبب خسائر للنبات بالكامل أو أجزاء

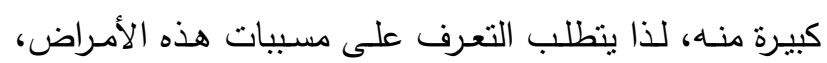

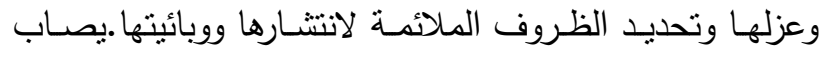

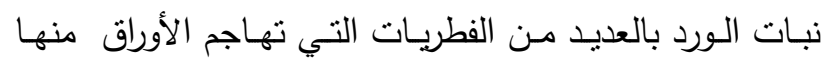

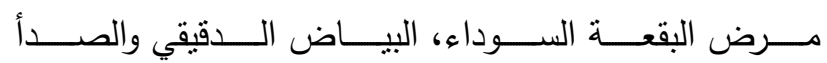
(Schwer 2006)، ويعد مرض البياض الدقيقى المتسبب عن إضن الفطري الاسكي Sphaerotheca pannosa var.rosae Podosphaera rosae مرض الصدأ الناتج عن فطر Phragmidium الذي يظهر

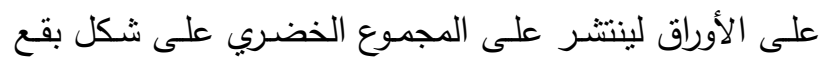

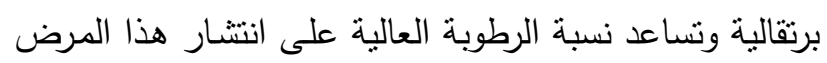

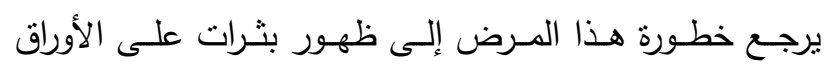

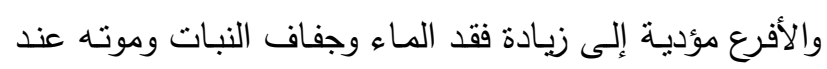
الإصابة الثديدة، أما مرض البقع السوداء الذي نظهر أعراضده

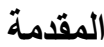

الورد Rose الاسم اللاتيني (Rosa sp.) هو نبات زينة للحدائق المنزليـة، والمسطحات ويُنَسَّى لأسباب تجاريـة ، فهو

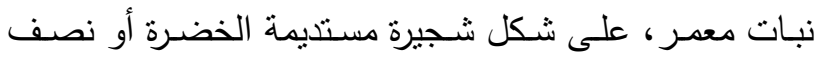

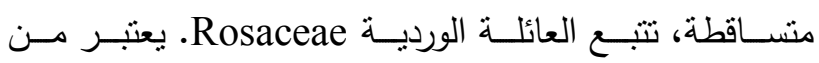

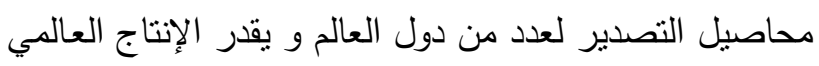

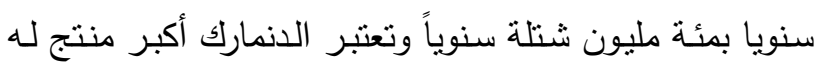

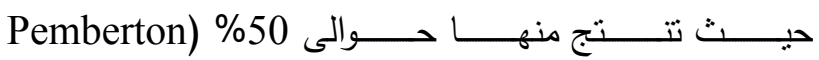
وآخرون، 2003). يمتاز هذا النبات بأنه متعدد الاستعمالات؛

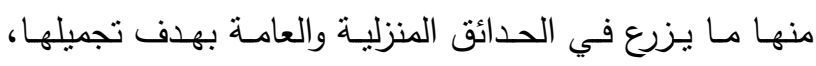

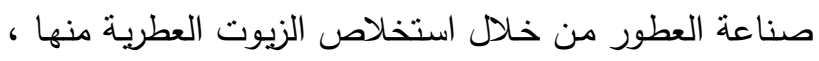
ويدخل في الصناعة العلاجية. نبات الورد يصاب بالعديد من الممرضات النباتية التي ينجم

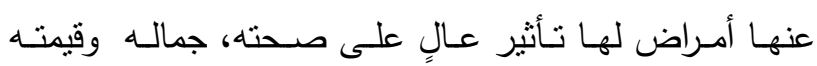


وارتفاع 624 متراً عن سطح البحر، تمتـاز بمنـاخ البحر

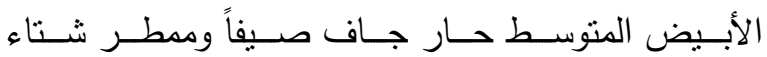
(Abdullrahman) خمسـة مواقـع لكل مـن : مشـاتل، حـدائق عامـة وحـدائق منزلية، خـلال فترة نشاط النبات متضمنة المواسم الأربعة مونة (الخريف، الثتاء، الربيع والصيف)، بمعدل خمس أثتجار

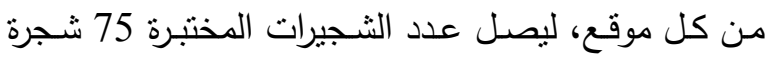
|ثـهر ، جلبت العينـات إلى معمل أمراض النبات، الكائن

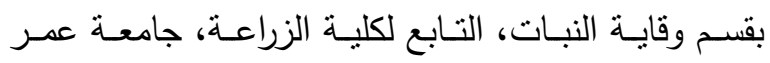
المختار ، البيضاء، لبييا بعد حفظها في صناديق بلاستيك ونه

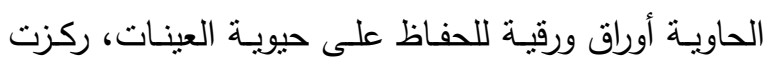
هذه الدراسـة على مواقع تختلف في طبيعـة الزراعـة بهـا

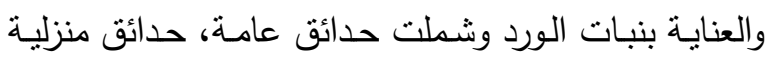

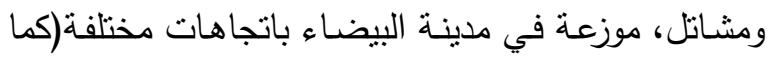
هو مبين بالخريطة )، وجمعت العينات من ثنلاثة مستويات

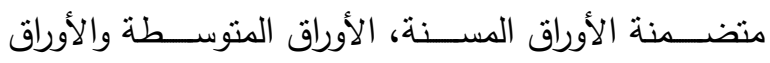

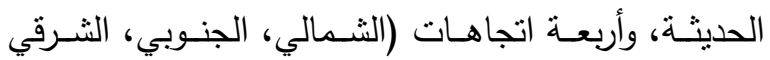
والغربي) لكل شجرة. تم الجمع بمعدل شهري في فترة نشاط واط التا

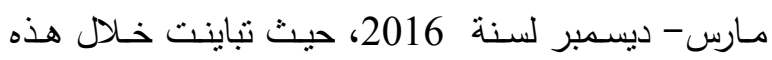
الفترة ظروف بيئية مختلفة نتيجـة تعاقب الفصول الأربعـة

$$
\text { (الخريف، الشتاء، الربيع والصيف). }
$$

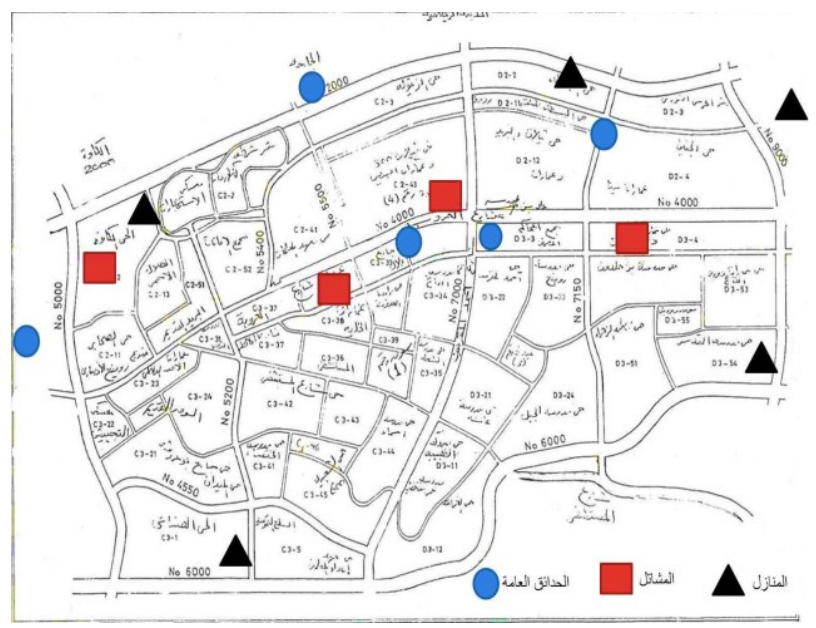

شكل ( 1) خريطة تبين موقع الدراسة
بوضـوح على شـكل بقع سـوداء على السـطح العلـوي للورقـة

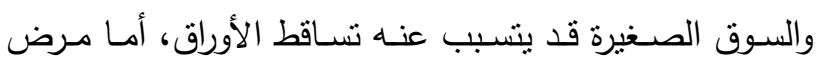

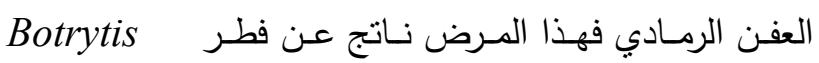
cinerea

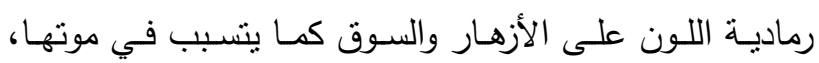
بالإضافة إلى مرض البياض الزغبي نظهر بقع أرجوانية اللون

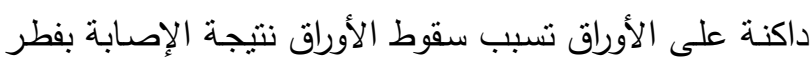
Pernospora sparsa Coryneum ‘Cercospora puderi ‘Alternaria sp ‘Mycosphaerlla rosicola ‘microstictum Salamone (Septoria rosae ذوآخرون،2009) أن (Podosphaera pannosa (Wallr. Fr البياض الدقيقي) .Botrytis cinerea Pers. والعفن الرمادي ، de Bary)

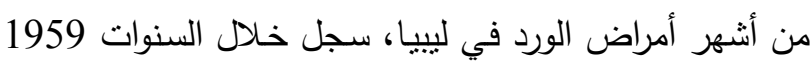
إلى 1964 في طرابلس العديد من الاصـابات على شجيرات

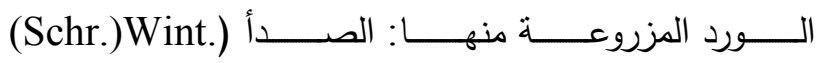
Subcorticium (Sphaerotheca pannosa (Waltas) Lev.)

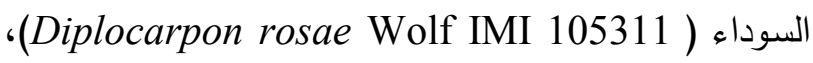
Botrytis cinerea Pers. Ex Fr. IMI ) والعفن الرمادي

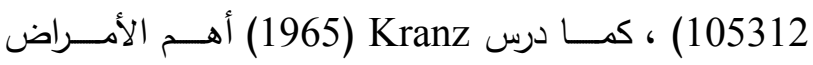

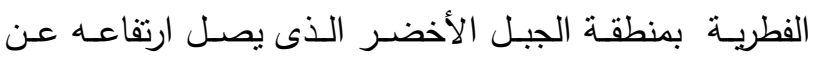
مستوى سطح البحر 800م، ومتوسط امطاره 200-600 مم 3

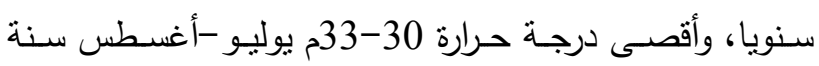
1959، وعزل فطر Gliocladium roseum من شجيرات الورد المزروعة بهذه المنطقة. تهدف هذه الدراسة إلى حصر أمراض الورد بمدينة البيضاء، الجبل الأخضر -ليبيا.

\section{المواد وطرق البحث}

جمع العينات: جمعت العينات من 15 موقعاً داخل مدينة البيضـاء الواقعـة في الثـمال الثـرقي مـن لبييـا، بالمنطقة

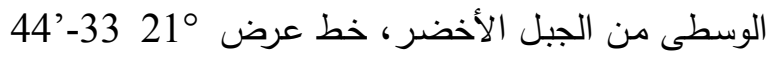

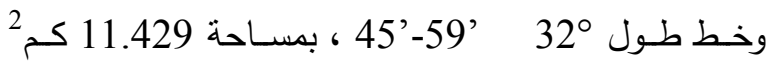


غير منتظم بني ووجود موت للحواف مع موت قمـة الورقة، (2-ب): يظهر عدد مـن التبقعـات بنيـة سـوداء صـغيرة

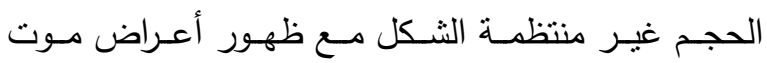
الحواف،(2-ج): بقع سوداء اللون مع موت الحواف، (2-

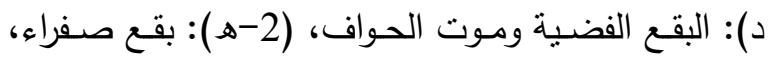
بثرات، مـوت الحواف، (2-و) : لطخـة وبقع سـوداء، (2-

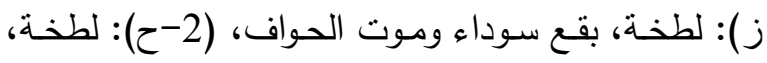
بقعة فضية، بقع سوداء وبقع محاطة بهالة صفراء، (2ط): مـوت القــة وبقـع فضـية، (-ي): بقـع فضـية وبقـع سوداء.

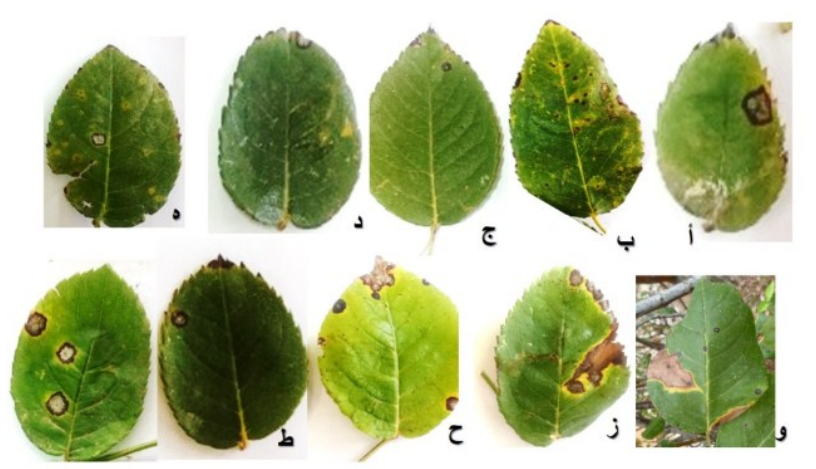

شكل:(2). أهم الأعراض الظاهرة على أوراق الورد خلال موسم نموها لسنة 2016م.

حصر أمراض الـورد المسـجلة بالاراسـة: يظهر من الجدول

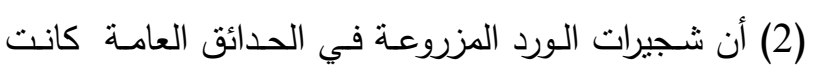

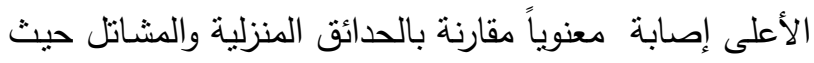

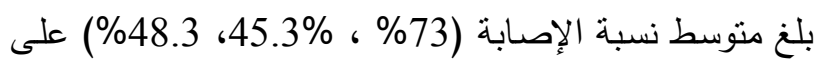
التوالي، وكانت نسـب الإصـابة بشكل عـام عاليـة طيلـة فترة الدراسة حيث وصلت أقل نسبة إصابة سجلت بسنة 2016 م 49.2\%، في حين أعلى نسبة إصابة على الورد سجلت في وبله

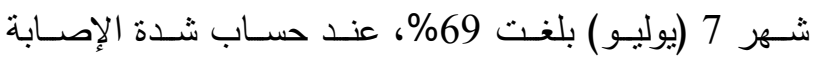

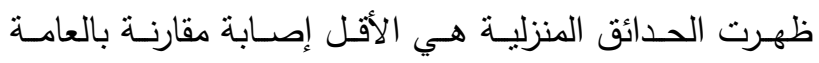
والمشاتل، وبينت النتائج أن الثهور الأعلى حرارة تراوحت شدئ الثدة الإصـابة بها 30-38\%، في حين كانـت شـهور الربيـع لـم

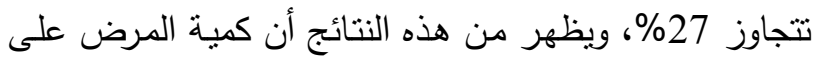

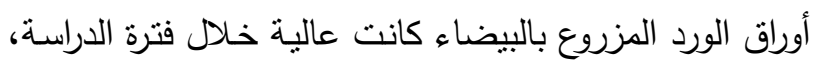

جدول:(1). المتوسطات الثهرية لعناصر المناخ بمحطة البيضـاء لسنة 2016

\begin{tabular}{|c|c|c|c|c|c|c|c|}
\hline \multicolumn{7}{|c|}{ العناصر المناخية* } & \multirow[b]{2}{*}{ الأشهر } \\
\hline 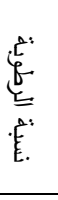 & 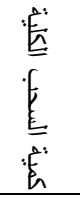 & 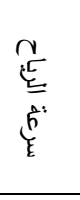 & 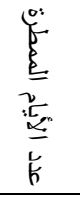 & 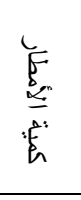 & $\frac{b}{\underline{E}}$ & $\frac{\underline{b}}{\underline{E}}$ & \\
\hline 72 & 05.0 & 11.0 & 06.0 & 04.0 & 06.2 & 14.9 & 3 \\
\hline 65 & 03.2 & 08.8 & 03.0 & 01.5 & 08.9 & 16.4 & 4 \\
\hline 61 & 01.0 & 09.0 & 02.0 & 0.2 & 10.5 & 17.5 & 5 \\
\hline 55 & 01.0 & 10.5 & 0 & 0 & 12.8 & 19.5 & 6 \\
\hline 50 & 0.5 & 10.5 & 0 & 0 & 14.6 & 22.0 & 7 \\
\hline 45 & 0.5 & 09.0 & 0 & 0 & 17.5 & 24.8 & 8 \\
\hline 46 & 01.0 & 09.0 & 02.0 & 0.9 & 20.0 & 28.0 & 9 \\
\hline
\end{tabular}

تقدير كميـة الإصـابة في المواقع : قيمت كميـة الإصـابة على أوراق نباتات الورد المجموعـة مـن كل موقع وكانت : لإنـ

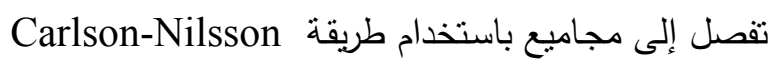
م \& Davidson (2002). مجاميع حسب المرض. تقدير كميـة الإصـابة بـالأمراض : قدرت أعراض الإصـابة

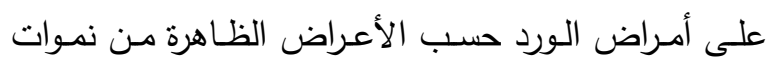

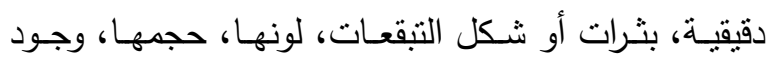

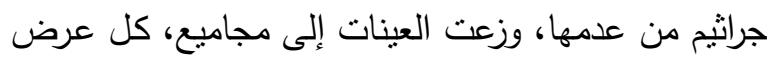
تم توزيعه حسب درجة الإصـابة لكل عرض وفق المقاييس

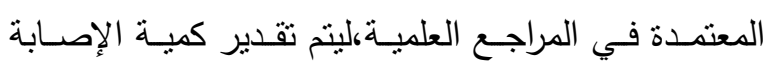
وحساب نسبة وشدة الإصابة لكل مرض على حدة. النتائج

أمراض الورد المسجلة بالاراسـة: من أهم النتائج المسجلة في هذه الدراسة هو ظهور العديد من الأعراض على الورقة الهم الوردة

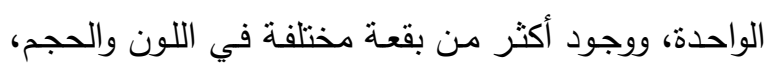

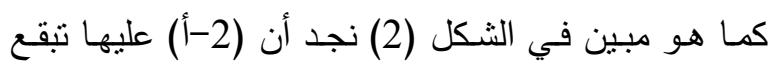




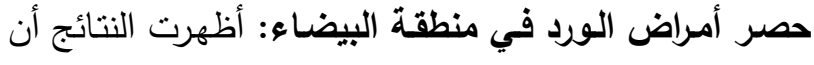

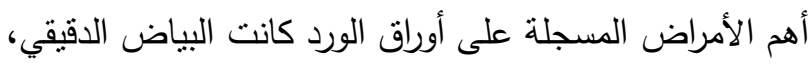

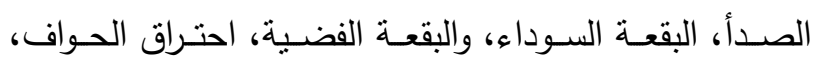

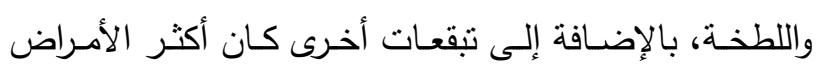

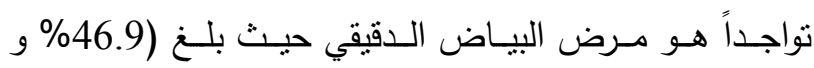

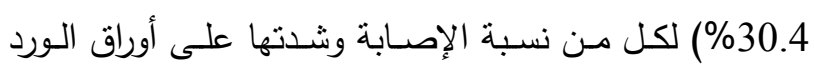

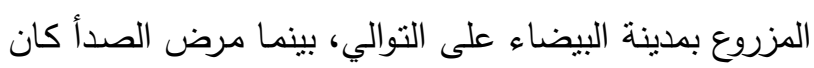

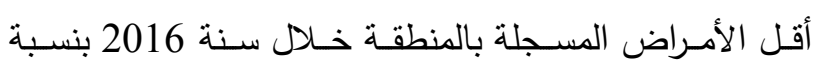

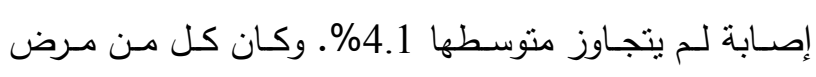
اللطخة ، البقعة الفضية، والبقعة السوداء عالية الإصابة مقارنة

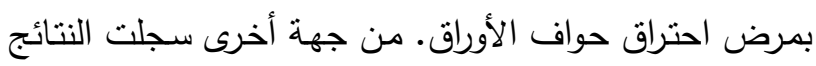

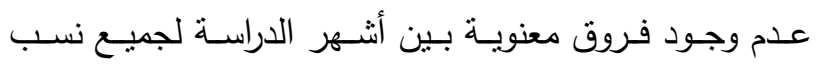

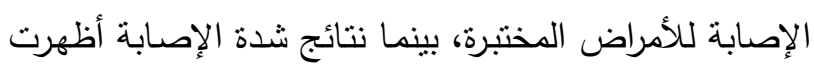

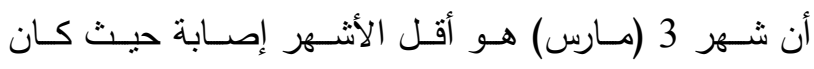
متوسطه 11.1\%، في حين ارتفعت شـدة الإصـابة خـلال

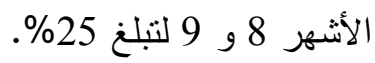

كانت الحدائق المنزلية والمشاتل أقلها أصابة مقارنة بالحدائق

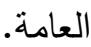
جدول (2) نسبة إصابة أوراق الورد المزروعة بحدائق البيضاء العامة والمنزلية ومشاتلها في الفترة الممندة (03-09 لسنة المرنة 2016م).

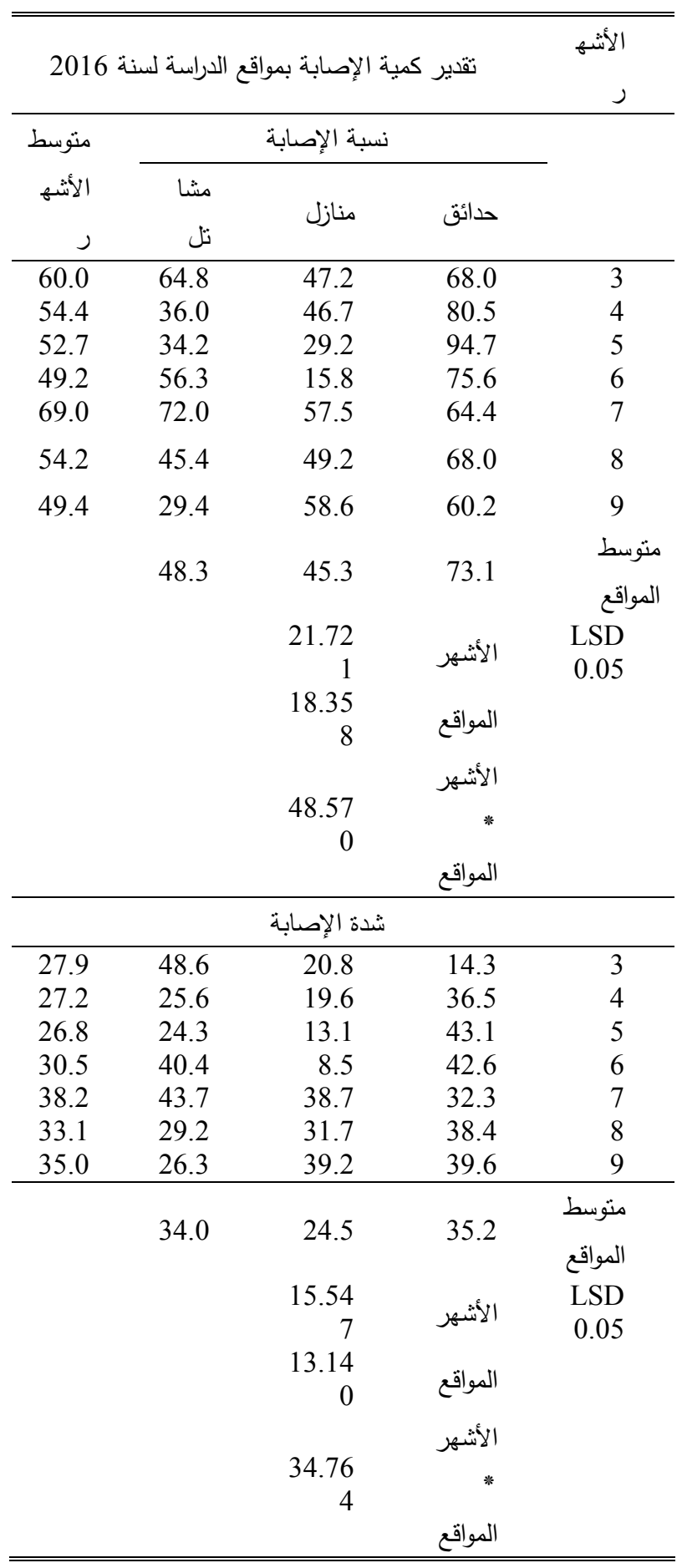


جدول ( 3) : نسبة الإصابة بأهم الأمراض الفطرية على أوراق الورد وشدتها خلال أشهر الدراسة لسنة 2016

\begin{tabular}{|c|c|c|c|c|c|c|c|}
\hline \multicolumn{7}{|c|}{ كمية الأمراض على أوراق الورد خلد الفترة (03-2016/09) } & \multirow{3}{*}{ 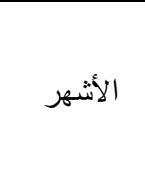 } \\
\hline \multicolumn{7}{|c|}{ 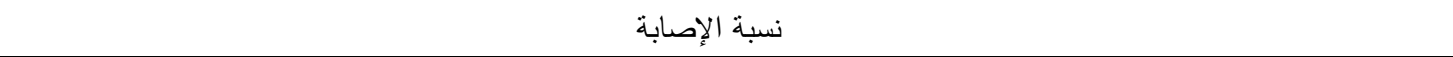 } & \\
\hline المتوسط & الصدأ & احتراق الحواف & البقعة السوداء & البقعة الفضية & اللطخة & البياض الدقيقي & \\
\hline 20.2 & 0.0 & 5.9 & 0.0 & 64.0 & 18.0 & 33.0 & 3 \\
\hline 37.0 & 6.2 & 20.7 & 70.2 & 60.5 & 28.9 & 35.5 & 4 \\
\hline 30.8 & 15.9 & 25.2 & 31.0 & 33.7 & 39.9 & 39.0 & 5 \\
\hline 27.3 & 0.0 & 30.0 & 22.9 & 21.5 & 43.8 & 45.3 & 6 \\
\hline 32.7 & 0.0 & 33.5 & 26.2 & 16.4 & 58.0 & 61.8 & 7 \\
\hline 35.4 & 0.0 & 19.9 & 32.8 & 40.4 & 63.4 & 56.1 & 8 \\
\hline \multirow[t]{5}{*}{34.5} & 6.9 & 10.4 & 38.6 & 37.6 & 55.6 & 57.7 & 9 \\
\hline & 4.1 & 20.8 & 31.7 & 39.2 & 43.9 & 46.9 & المتوسط \\
\hline & & & & 12.497 & \multicolumn{2}{|c|}{ الأمراض } & LSD 0.05 \\
\hline & & & & 11.570 & \multicolumn{2}{|c|}{ الأثهر } & \\
\hline & & & & 30.612 & \multicolumn{2}{|c|}{ الأمراض* الأشهر } & \\
\hline \multicolumn{8}{|c|}{ شدة الإصابة } \\
\hline 11.1 & 0.0 & 5.9 & 0.0 & 29.0 & 10.0 & 21.6 & 3 \\
\hline 22.4 & 6.2 & 16.5 & 30.8 & 37.4 & 18.4 & 25.1 & 4 \\
\hline 22.8 & 12.1 & 19.0 & 24.7 & 29.7 & 22.6 & 28.5 & 5 \\
\hline 20.9 & 0.0 & 20.4 & 22.9 & 19.8 & 29.9 & 32.5 & 6 \\
\hline 20.6 & 0.0 & 26.2 & 15.6 & 17.7 & 30.3 & 33.6 & 7 \\
\hline 25.8 & 0.0 & 18.7 & 27.3 & 39.1 & 33.0 & 36.4 & 8 \\
\hline \multirow[t]{5}{*}{25.7} & 6.1 & 7.9 & 34.8 & 37.5 & 32.5 & 35.3 & 9 \\
\hline & 3.5 & 16.4 & 22.3 & 30.0 & 25.2 & 30.4 & المتوسط \\
\hline & & & & 7.84 & \multicolumn{2}{|c|}{ الأمراض } & LSD 0.05 \\
\hline & & & & 7.26 & \multicolumn{2}{|c|}{ الأشهر } & \\
\hline & & & & n.s & \multicolumn{2}{|c|}{ الأمراض* الأشهر } & \\
\hline
\end{tabular}

أظهرت نتائج الدراسـة المبينة بالثكل (3) أن العلاقة بين

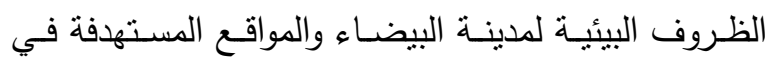

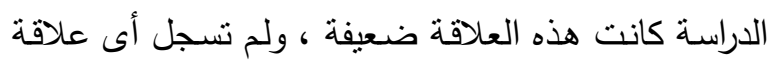

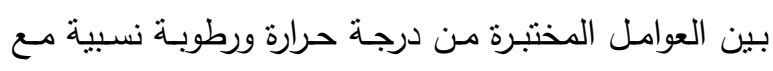

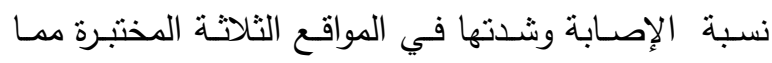

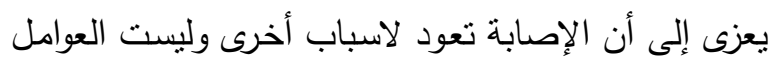
البيئية فقط. 


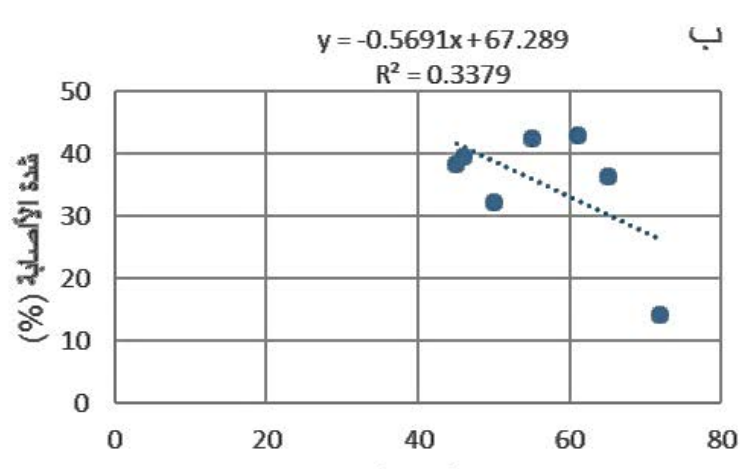

الرطوبة الثنيبة (\%)

د

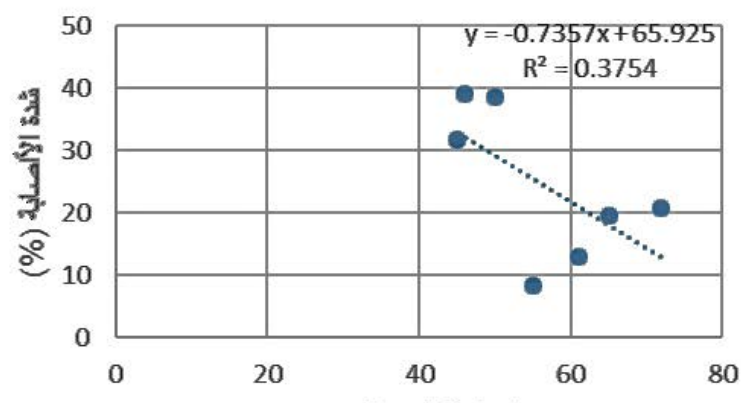

الرطوبة الثمبية (\%)

$$
\begin{gathered}
y=0.282 x+18.142 \\
R^{2}=0.0828
\end{gathered}
$$

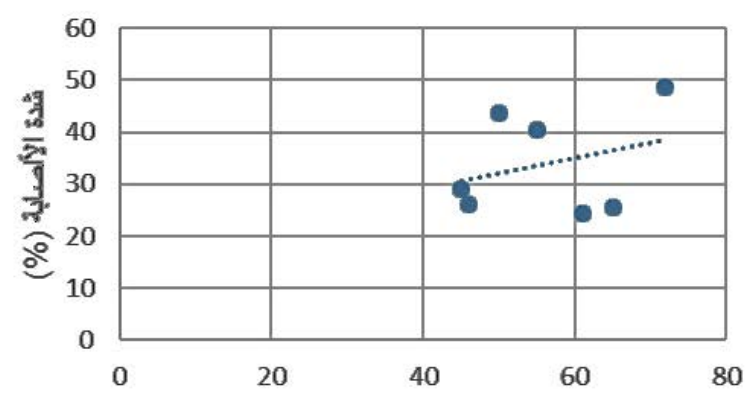

الرطوية الثمبية (\%)

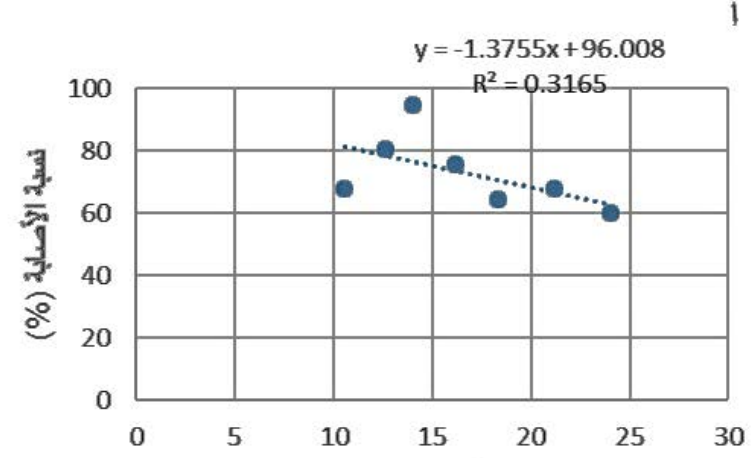

(م) مجة الحرارة

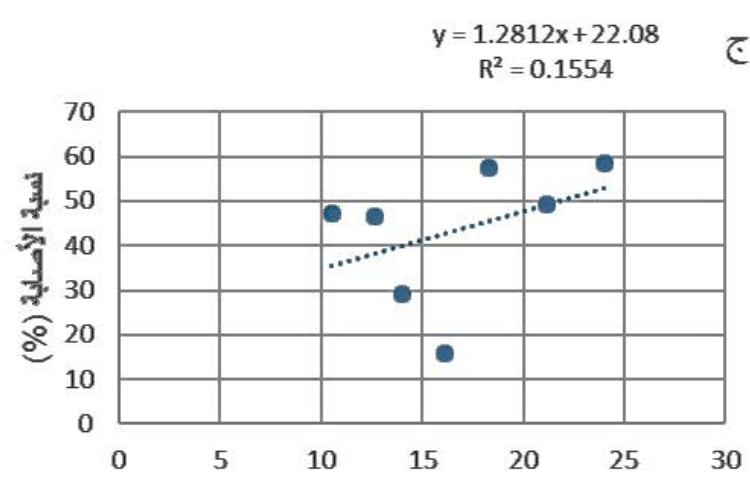

(م) - مجة الصرارة

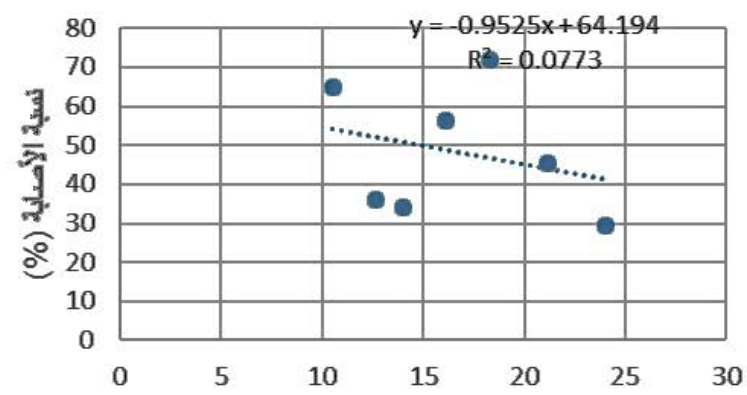

(م) - مجة الحرارة

شكل (3) :العلاقة بين الظروف البيئية (درجة حرارة ورطوبة نسبية مع كمية الأمراض الفطرية في المواقع الثلاثة: ( أ ، ب) نسبة الإصابة وشدتها في

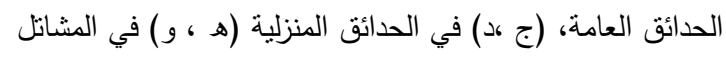

الذي كان أعلى الفصول أصابة، بينما كانت هناك فروق غير معنويـة بين فصلي الصيف والخريف في حين سجل

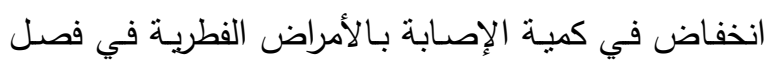
أظهرت نتائج الدراسـة المبينة بالثكل (4) تسجيل أمراض الثتاء.

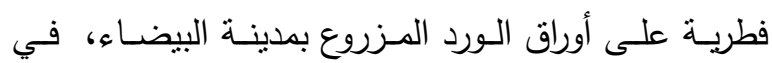
جميع فصول سنة 2016 المتعاقبة، وبخاصـة فصل الربيع 

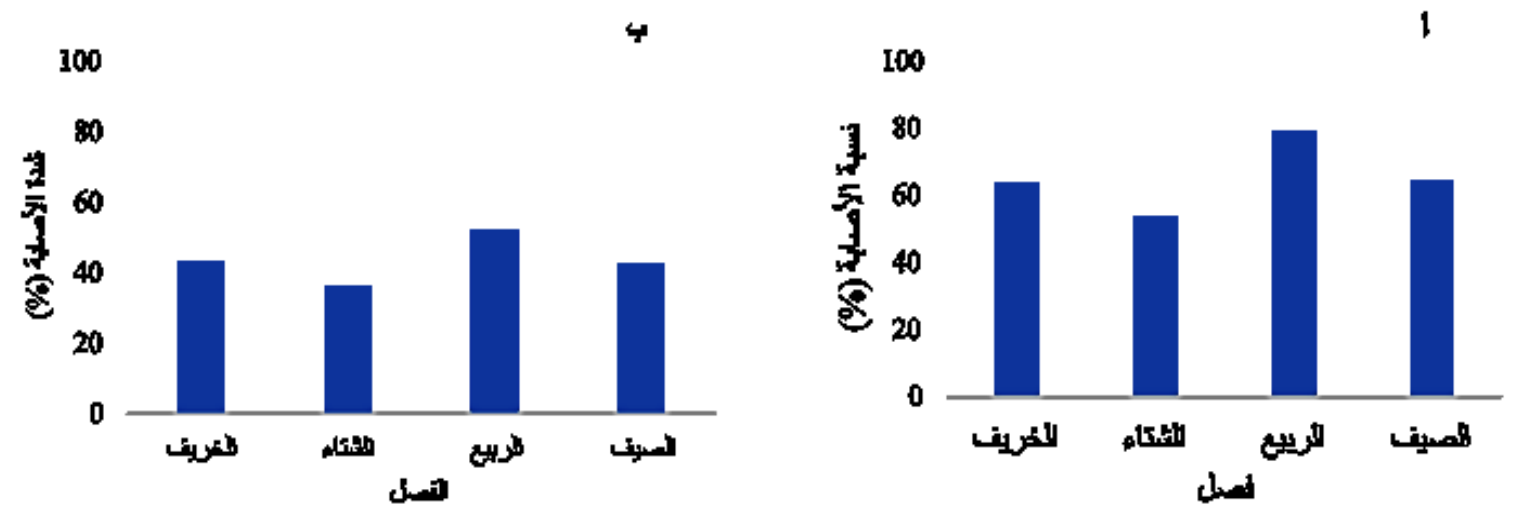

شكل ( 4): نسبة الإصابة بأمراض الورد في منطقة البيضاء وشدتها خلال المواسم الأربعة (أنسبة الإصابة، ب: شدة الإصابة)

أنشهر الصيف وتصل أعلى أصابة في شهري يونيو ويوليو

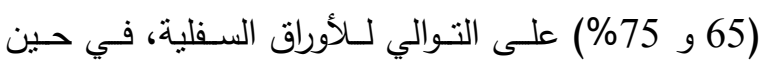
كانت أثشر الربيع والخريف هي الأقل أصابة.

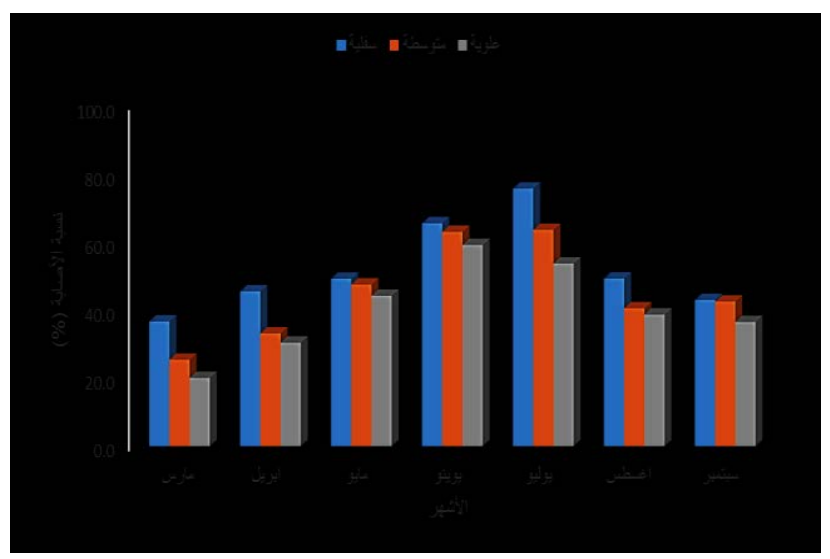

شـكل (5) تأثنر المستويات على أمـراض الورد في منطقة البيضـاء خلا أشهر الدراسة

\section{المناقشة}

تم في هذه الدراسة التعرف على الأمراض التى تهاجم أوراق

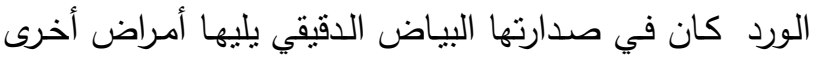

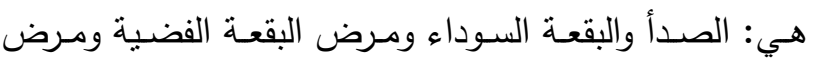
موت الحواف واللطخة، وقد سجلت هذه الدراسة تواجد مرض البه

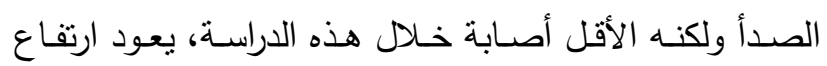
الإصابة البياض الدقيقي إلى عدة عوامل متداخلة كالظروف

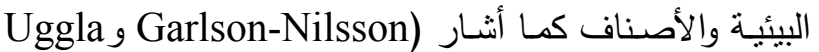

وعند حسـاب نسبة الإصـابة بـالأمراض الفطريسة في الاتجاهـات

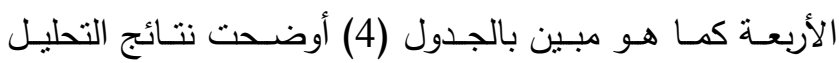
الإحصـائي عدم وجود فروق معنويـة بين الاتجاهـات وأن نسبة بـانة الإصـابة تتراوح بين 37.5-40\%، وكان شـهر يوليو هو أعلى بروف

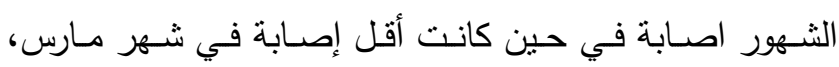
ويلاحظ أن أنشهر الصيف تجاوزت بها نسبة الإصابة 50\% جدول (4) تأثير الاتجاهات على نسبة الإصـابة بالأمراض الفطريـة على أوراق الورد في منطقة البيضاء خلا أثنهر الدراسة الآنا

\begin{tabular}{|c|c|c|c|c|c|}
\hline \multirow{2}{*}{\multicolumn{5}{|c|}{ نسبة الإصابة (\%) بالأمراض الفطرية على أوراق الورد حسب }} & \multirow{3}{*}{ الأشهر } \\
\hline & & & & & \\
\hline المتوسط & جنوب & شمال & غرب & شرق & \\
\hline 23.3 & 25.0 & 18.8 & 25.9 & 23.3 & مارس \\
\hline 36.4 & 32.0 & 41.6 & 31.3 & 40.8 & ابريل \\
\hline 39.9 & 43.6 & 33.9 & 40.4 & 41.6 & مايو \\
\hline 51.2 & 52.5 & 45.9 & 58.7 & 47.8 & يوينو \\
\hline 55.1 & 53.4 & 58.2 & 53.3 & 55.7 & يوليو \\
\hline 34.5 & 36.5 & 34.8 & 28.9 & 37.7 & اغسطس \\
\hline \multirow[t]{2}{*}{31.0} & 37.2 & 29.2 & 30.4 & 27.2 & سبتمبر \\
\hline & 40.0 & 37.5 & 38.4 & 39.2 & المنوسط \\
\hline
\end{tabular}

أظهـرت نتـائج الدراســة المبينــة بالثــكل (4) أن الأوراق المسنة الواقعة في الجزء السفلي من الفرع هى الأعلى نسبة

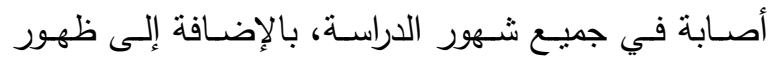
اختلاف معنوي بين أثنهر الدراسة حيث تزداد الإصابة في 
نتائج الدراسـة على وجود علاقـة بين عمر الورقـة وكميـة

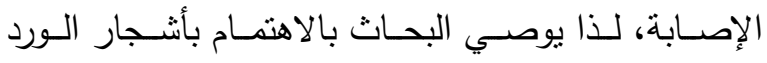

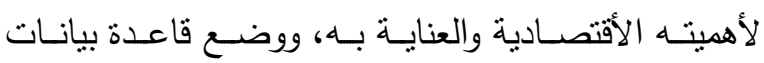
للفطريات الممرضة للورد.

\section{المراجع}

عبداله، نوال جمعة (2019). دراسة الأمراض الفطرية. على أوراق الورد النامي بمدينة البيضاء. رسالة ماجستير ، غيـر منشـورة، قسـم وقايـة النبـات، كليـة الزراعة، جامعة عمر المختار، البيضاء، ليبيا.

Carlson-Nilsson, B. U. (2002). Variation in Rosa with emphasis on the improvement of winter hardiness and resistance to Marssonina rosae (Blackspot). Swedish University of Agricultural Sciences.

El-Gali, Z., \& Abdullrahman, E. (2014). Distribution of some molds in the atmospheric air of El-Beida city, Libya. International Journal of Innovative and Applied Research, 6, 1-7.

Kranz, J. (1965). A list of plant pathogenic and other fungi of Cyrenaica (Libya). Phytopathological Papers(6).

Pemberton, H., Kelly, J., \& Ferare, J. (2003). Pot rose production. Pp: 587-593. Encyclopedia of rose science. Academic press. Oxford, USA. 1450p. http://dx. doi. org/10.1016/B0-12227620-5/00074-4.

Salamone, A., Scarito, G., Scovazzo, G. C., \& Fascella, G. (2009). Control of Powdery Mildew in Cut Roses using Natural Products in the Greenhouse. Floriculture and Ornamental Biotechnology, 3, 121-125.
، 2005) كمــــــــ الاخـتلاف بـين الأصــاف إلـى وجـود حواجز ميكانيكية وكيميائية تمثلت في نراكم المواد الفينولية عند مواقـع الإصـابة ومنـاطق دخـول المسـبب (Schwer2006) أظهرت النتائج أن الإصابة بالأمراض الفطرية على أوراق الورد

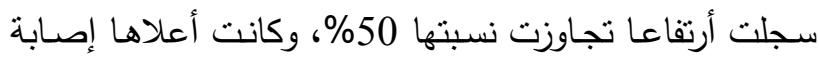

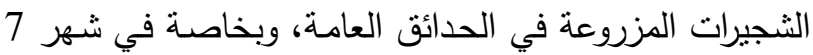
(يوليو) حيث بلغت نسبة الإصـابة( 69\%)، في حين كانت الحدائق المنزلية والمشـاتل أقل أصـابة. كما يتضـح من الإدنائج

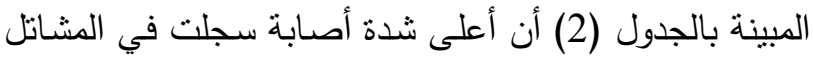
بلغت 48.6\% في شهر مارس إلا أن هذه النسبة تناقصت في الأشهر اللاحقة ويعزى سبب هذا الانخفاض إلى أن المشاتل تزداد بها العنايـة والاهتمـام وتطبيق برامج المكافحـة للحصـول

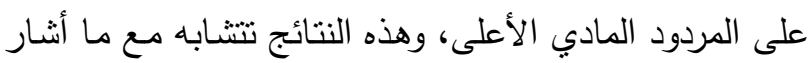

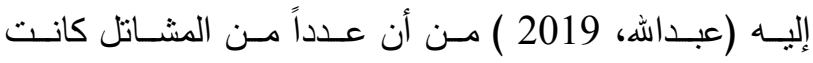

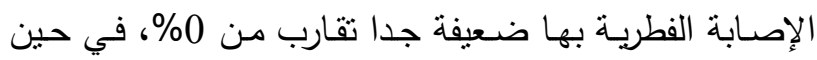
بينت نتائج الدراسة أن الحدائق العامة كانت شدة الإصابة بهابها 14.3 في شهر مارس إلا أن الإهمال أدى إلى ارتفاع كمية

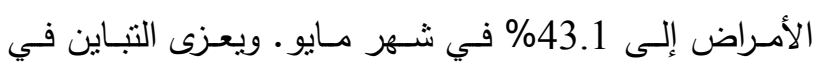

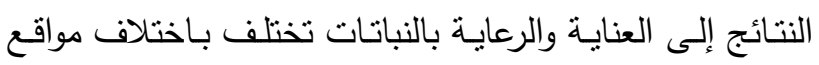

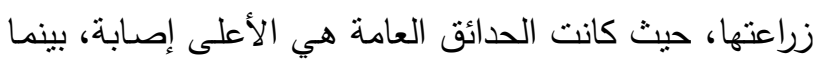

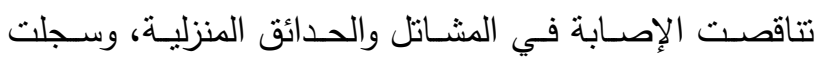
الدراسـة عدم وجود اختلاف بين الجهات الأربعة للشجرة، في

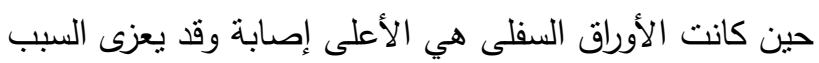
إلى ضعف مقاومة الورقة المسنة.

من هذه الدراسـة نسنتتج أنـهـ لم يسـل مرض منفرد على

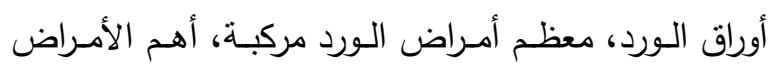
المسجلة على أوراق الورد هي: البياض الدقيقي، الصدأ،

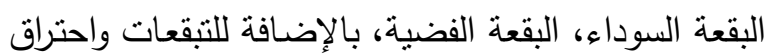
الحواف، وموت قمة الورقة واللطخة، سجل تباين بين مواقع

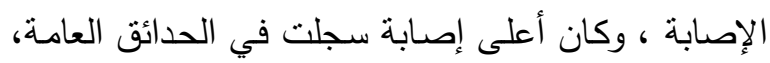

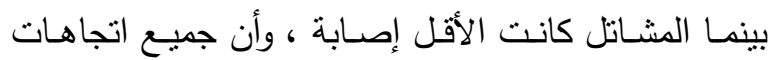

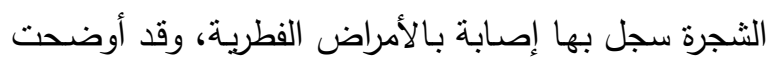


مجلة الدختار للعلوم 34 (4): 372-381، 2019

Schwer, C. S. (2006). Impact of foliar fungi on dogroses. SLU, Dept. of Crop Science.

Uggla, M., \& Carlson-Nilsson, B. (2005). Screening of fungal diseases in offspring from crosses between Rosa sections Caninae and Cinnamomeae. Scientia Horticulturae, 104(4), 493504. 


\title{
Survey of Some Fungal Diseases on Leaves of Rose Plants in Al-Bayda City, Libya
}

\author{
Nwara A. Mohamed*, Nawal G. Abdullah and Omar M. El-Sanousi \\ Plant protection Department, Agriculture Faculty, Omar Al-Mukhtar University, Al-Bayda, \\ Libya
}

Received: 13 June 2019/ Accepted: 30 December 2019

Doi: https://doi.org/10.54172/mjsc.v34i4.235

\begin{abstract}
This study was conducted on rose plants growing in Al-Bayda City's in 2016 to survey the fungal diseases on leaves of this plant. The results of this study indicated that the infection on roses planted in public gardens (parks) was more significant than that of home or nursery planted roses, and these results showed that the amount of disease on leaves of roses grown in El-Beida were high during the period of this study, Home gardens and nurseries were the least infected $(45.5 \%$ and $48.3 \%)$ respectively, compared to parks $(73 \%)$. The reported diseases in this study were powdery mildew, rust, black spot, silver spot, burning margins and blotches, the most disease occurring was powdery mildew disease at $(46.9 \%$ and $30.4 \%$ ) for both incidence rate and severity respectively on cultivated roses in El-Bieda City, While rust was the least registered disease during the year 2016 with an infection rate that did not exceed an average of $4.1 \%$. There are no significant differences between the trends and incidences of infection range between $37.5-40 \%$. July was the highest month in terms of infection and the older leaves of roses had the highest infection rate through all the months of our study.
\end{abstract}

Keywords: Survey, fungal diseases, rose leaves, Al-Bayda city, Libya. 